\title{
Removing Stripes, Scratches, and Curtaining with Non-Recoverable Compressed Sensing
}

Jonathan Schwartz ${ }^{1}$, Yi Jiang ${ }^{2}$, Yongjie Wang ${ }^{3}$, Anthony Aiello ${ }^{3}$, Pallab Bhattacharya ${ }^{3}$, Hui Yuan ${ }^{4}$, Zetian $\mathrm{Mi}^{3}$, Nabil Bassim ${ }^{5}$ and Robert Hovden ${ }^{1}$

1. Department of Materials Science and Engineering, University of Michigan, Ann Arbor, MI, USA.

2. X-ray Science Division, Advanced Photon Source, Argonne National Laboratory, Argonne, IL, USA.

3. Department of Electrical Engineering and Computer Science, University of Michigan, MI, USA.

4. Canadian Centre for Electron Microscopy, McMaster University, Hamilton, ON, Canada.

5. Department of Material Science and Engineering, McMaster University, Hamilton, ON, Canada.

Highly-directional image artifacts such as ion mill curtaining, mechanical scratches, or stripes from beam instability degrade the interpretability of micrographs [1]. These unwanted, aperiodic features extend the image along a primary direction and occupy a small wedge of information in Fourier space. Deleting this wedge of data replaces stripes, scratches, or curtaining, with more complex streaking and blurring artifacts - known within the tomography community as 'missing' wedge artifacts. [2]. Here, we overcome this problem by recovering the missing region using total variation (TV) minimization, which leverages image sparsity-based reconstruction techniques - colloquially referred to as compressed sensing-to reliably restore images corrupted by stripe-like features. Our approach removes stripe features (i.e. beam instability, ion mill curtaining, mechanical scratches) and remains robust at low signal-to-noise [3].

Here we destripe images, by first removing the information in Fourier space containing unwanted artifacts, thus creating a 'missing' wedge. This 'missing' wedge of information is then recovered by minimizing the image's TV. Simply deleting information within the 'missing' wedge in Fourier Space (Figure 1b.) provides a poor estimate of the clean image and creates elongation and blurring artifacts perpendicular to the 'missing' wedge (Fig. 1b). The residuals reveal the quality of the decomposition by calculating the absolute difference between the output and the original image. The residuals from a wedge Fourier filter, for Figure 1b, shows the removal of both unwanted stripes and useful structural information (Fig. 1d), a typical problem associated with filtering. However, recovering information in the 'missing' wedge with TV minimization produces an image without these artifacts (Fig. 1c). Figure 1e shows TV minimization only removed features that strictly pertain to the scratches [3].

To emphasize the adaptability of our algorithm, Figure 2a shows an atomic resolution bright field scanning transmission electron microscope (BF-S/TEM) image of an $\mathrm{Al}_{\mathrm{x}} \mathrm{Ga}_{1-\mathrm{x}} \mathrm{N}$ quantum well suffering from unidirectional horizontal stripes caused by current fluctuations in the beam. The stripes prevent assessment of interface sharpness of the bright Al-rich layer. The residual between the corrupted image and reconstruction (Figure 2c) shows that only unidirectional stripes are removed. Furthermore, our approach is robust even down to low signal to noise ratios (SNR) and has the capability to preserve fine features. For demonstration, our algorithm consistently produces results similar to the image in Figure $3 \mathrm{a}$ after random Gaussian white noise is added (Fig. 3b-c) [3].

To further understand the relationship between SNR and the missing wedge, Figure $3 \mathrm{~d}$ shows a plot of the reconstruction's root mean square error (RMSE) normalized by the RMSE of the images before reconstruction (i.e. with missing wedge). The results show that our approach best reconstructs the test object for small missing wedges $\left(\$ 12^{\circ}\right)$ and SNR values above 10 [3]. Ultimately, we demonstrate a CS- 
based approach to remove highly directional features without introducing new artifacts. The code is published and available open-source (BSD license) through tomviz.org

\section{References:}

[1] S Zaefferer, SWright and D Raabe, Metallurgical and Materials Transactions A 39 (2008), p. 374.

[2] R Leary, Z Saghi and P Midgley, D. Holland Ultramicroscopy 131 (2013), p. 70.

[3] J Schwartz et al., arXiv:1901.08001 [cs.CV].

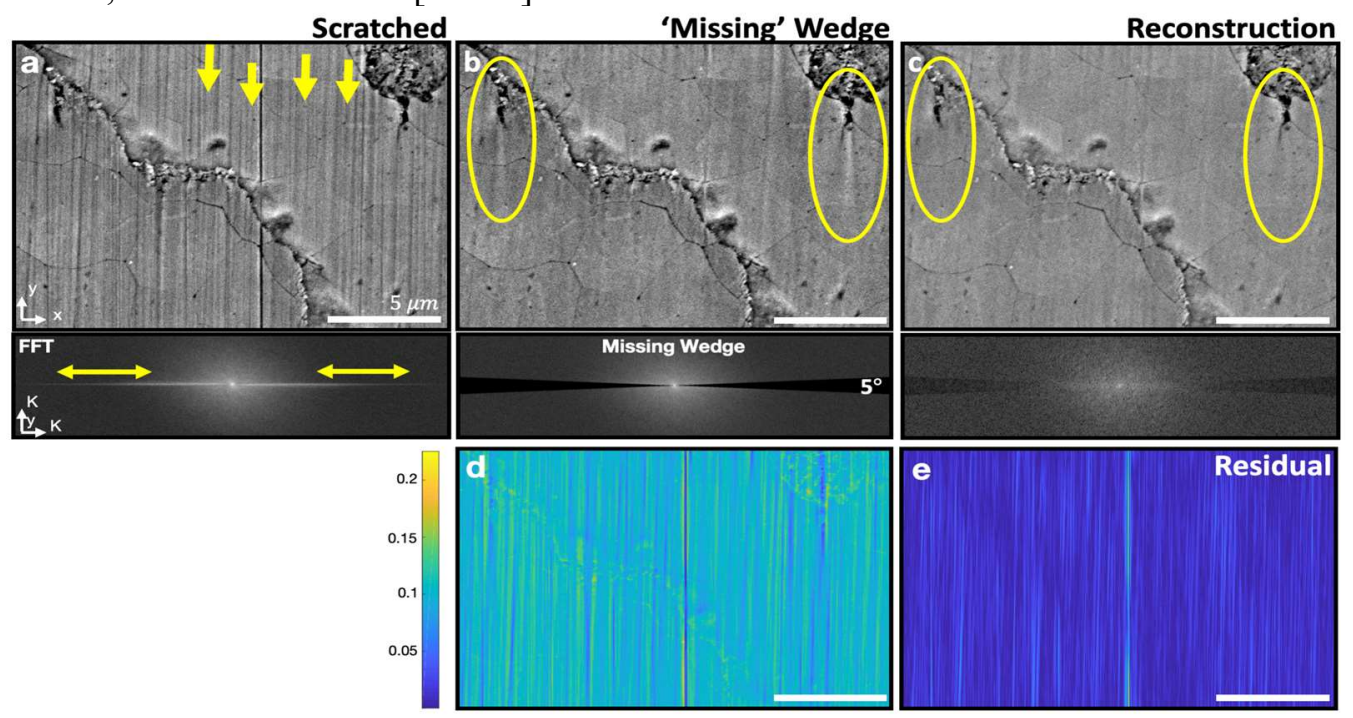

Figure 1. Reconstruction of a scratched pearl surface. a, The original backscatter electron SEM image of a scratched biomaterial with its FFT below. b, The reconstruction by deleting a wedge of information in Fourier space. c, The TV minimization reconstruction. d-e, The Residual between images (b)/(a) and (c)/(a).
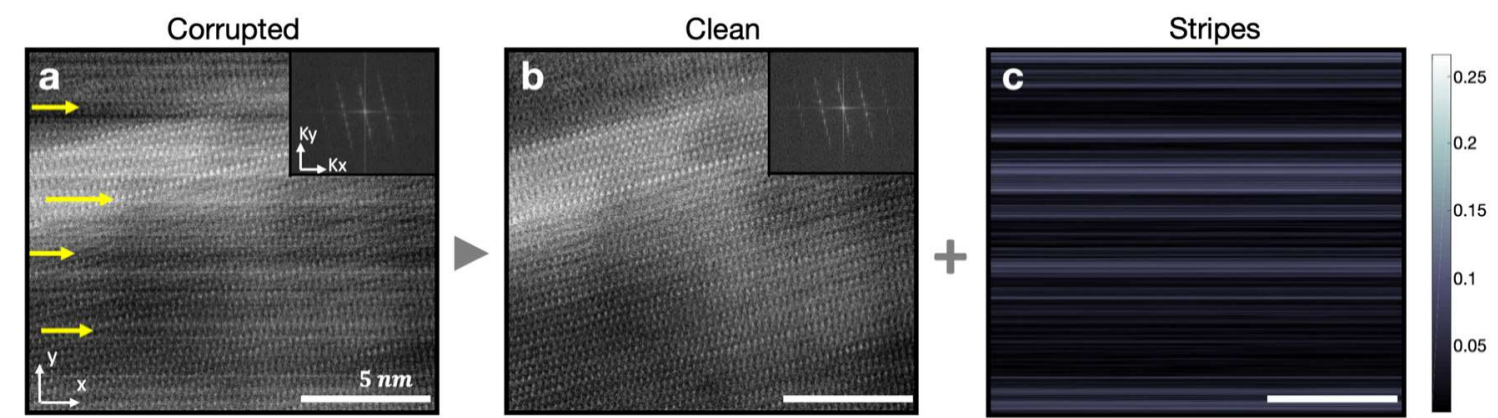

Figure 2. Reconstruction of an atomic Resolution S/TEM micrograph. a, The original BF-S/TEM image of an AlGaN Quantum Well with horizontal intensity fluctuations and its FFT on the top right-hand corner. To emphasize the resolution, the contrast is reversed. b, The TV minimization reconstruction and its FFT on the top right-hand corner. c, The residual between images (b)/(a).
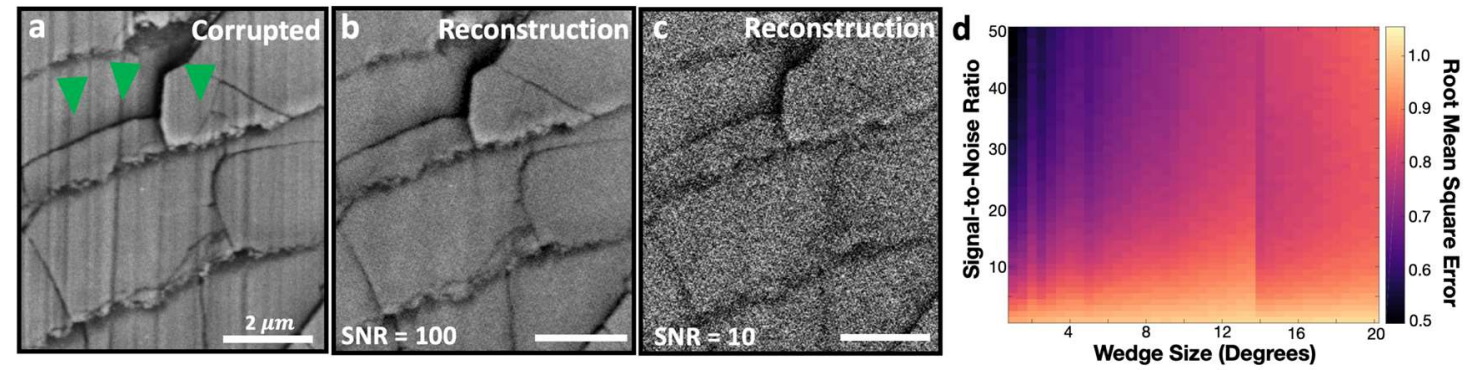

Figure 3. Reconstruction under multiple SNR. a-c, A secondary electron (SE)-SEM image of a scratched biomaterial reconstructed at different SNRs. d, A plot of the RMSE normalized by the error from various missing wedge sizes for a different HAADF-S/TEM micrograph. Values below 1 indicate the reconstruction outperforms loss of information. 